\section{Semiconductor X-Ray Detectors}

Rob Sareen

Gresham Scientific Instruments

Detection of characteristic $x$-rays is a fascinating and challenging subject. From its early beginnings with gas proportional counters it has evolved, like many branches of technology, into the use of a variety of semiconductors.

The lithium compensated silicon detector $[\mathrm{Si}(\mathrm{Li})]$ has been the predominant measuring tool over the last two decades. In the last five years, increasing numbers of high purity germanium detectors (HPG) have appeared and more recently a plethora of new materials and concepts are seeing a successful introduction. Among these newer materials are compound semiconductors like mercuric iodide, cadmium telluride, cadmium zinc telluride, gallium arsenide, lead iodide, indium phosphide and diamond. Among the new concepts are Bolometers, Transition Edge Detectors, Drift Detectors, PIN Diodes, CCD arrays and PN CCD arrays.

Except for the Bolometers and Transition Edge Detectors, all other detectors rely on certain basic principles like ionisation and depletion. The Bolometers measure the energy of the photon directly as a temperature increase and the Transition Edge Detectors are held in the super-conducting phase until the photon arrives. The energy of the photon causes sufficient thermal energy to be deposited to move the material into the resistive phase. Both these approaches rely on low temperatures, close to liquid helium.

This short article considers the theory of operation of the most extensively used class of semiconductor detectors - namely the Si(Li). In the early sixties, thin depleted silicon diodes were used to detect particles like electrons, protons, alpha particles, tritons and so forth. Depletion depths of over $100 \mu \mathrm{m}$ were possible and these could stop, for example, $3 \mathrm{MeV}$ protons. Nuclear physics research benefited enormously from this new detector which was small, insensitive to magnetic fields, capable of working for long periods in a vacuum and had excellent energy resolution (15 keV FWHM on $5.48 \mathrm{MeV}$ alpha particles). The range of preamplifiers, high voltage supplies and amplifiers that were designed for these detectors became the foundation for the technology used in the modern Si (Li) detector.

In parallel with this work, germanium detectors were developed for high-resolution gamma ray measurements. These again were to supplement the well-proven plastic scintillator and sodium iodide detectors that were in use with low dark current photo-multiplier tubes. Just like the silicon particle detector, the germanium gamma ray detectors were fundamental in the development of nuclear structure theory. Large volume detectors could

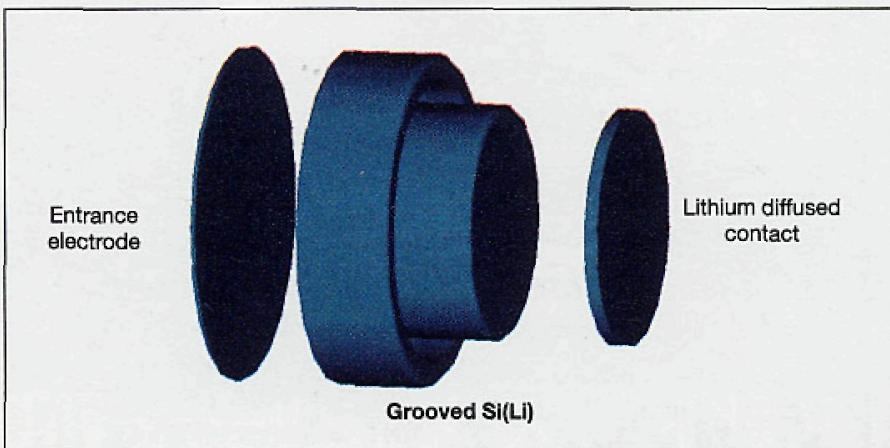

Fig 1: Silicon Cylinders are machined into a grooved structure to help with handling and reduce leakage currents.

\begin{tabular}{|llllll|}
\hline $\begin{array}{l}\text { Material } \\
\text { type }\end{array}$ & $\begin{array}{l}\text { Resistivity } \\
\text { Kohm-cm }\end{array}$ & Blas volts & $\begin{array}{l}\text { Depletion } \\
\text { depth } \\
\text { microns }\end{array}$ & $\begin{array}{l}\text { Highest energy } \\
\text { electron keV } \\
\mathbf{1 0 0 \%} \text { absorption }\end{array}$ & $\begin{array}{l}\text { Highest energy } \\
\text { X-Ray keV } \\
100 \% \text { absorption }\end{array}$ \\
\hline P Type & 10 & 100 & 300 & 270 & 7 \\
N-Type & 10 & 100 & 500 & 360 & 9 \\
\hline
\end{tabular}

efficiently measure gamma rays up to energies of several $\mathrm{MeV}$ with high precision (l.7 keV FWHM at $1.332 \mathrm{MeV}$ ). Similar electronic designs were required for both detectors. Consequently there was a wealth of technology available and a reasonable understanding of the theory and practice behind the operation of a semiconductor detector and its associated measuring electronics leading to the emergence of the family of $\mathrm{Si}(\mathrm{Li}) \mathrm{x}$-ray detectors.

\section{Silicon}

The silicon particle detector or "Surface Barrier" detector can be made from both $\mathrm{N}$ and $\mathrm{P}$ type silicon which should be pure with a resistivity preferably in the 1-20 Kohm-cm range and a carrier lifetime approaching $1 \mathrm{msec}$. By applying contacts to the opposite faces of a silicon slice, one an ohmic contact and the other a rectifying contact a PN junction can be formed. This can be depleted by the application of external bias. The approximate depletion depth relationships for the two types of silicon are:

$\begin{array}{ll}d=0.5(\rho V)^{1 / 2} & \text { for N-type silicon } \\ \text { and } & \text { for P-type silicon } \\ d=0.3(\rho V)^{1 / 2} & \end{array}$

where $\rho=$ material resisitivity in ohm-cm and $V$ =applied voltage

Obtaining higher resistivity silicon in regular quantities with good lifetimes and low dislocation densities has been difficult. Occasionally material of resistivity as high as $80 \mathrm{Kohm}-\mathrm{cm}$ has become available. Shallow depletion depths for stopping particles are sufficient but their efficiency on $\mathrm{x}$-rays is extremely low as table one shows. Here, the bias applied to the detector is 100 Volts, a practical upper value for a surface barrier detector before the onset of excess leakage current.

\section{X-Ray absorption in 3000 micron thick silicon}

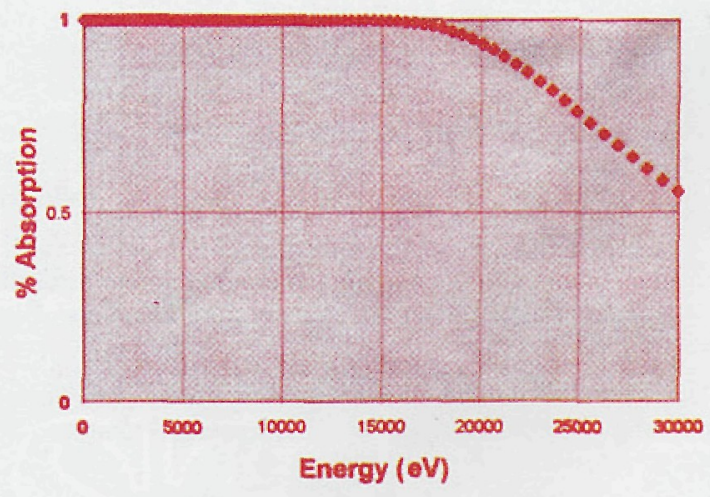

Fig. 2; Absorption of X-Rays in $3 \mathrm{~mm}$ silicon

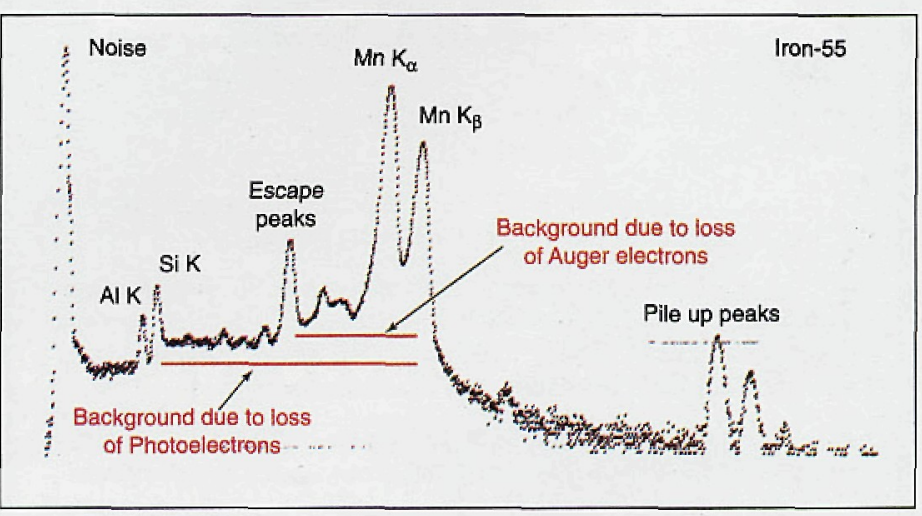

Fig.: 3: Fe 55 spectrum 


\section{Lithium Compensation (Lithium Drifting)}

To stop the more energetic $x$-rays, a deeper depletion depth is required. This can be achieved by using the technique called "Lithium Drifting" where lithium ions are made to move through P-type silicon and electrically neutralise the residual impurities. This compensation procedure achieves active depths of well above $3000 \mu \mathrm{m}$. The lithium ion is physically small and readily ionised at room temperature. When a layer of lithium is diffused into one face of a planar P-type silicon wafer, it makes an N-Type contact. The resulting PN junction can support an applied voltage. The lithium ions can be encouraged to move under the influence of the field at this junction. Increasing the crystal's temperature raises the mobility and so the drifting process usually is implemented at temperatures above $10,000^{\circ} \mathrm{C}$. As the ions move through the silicon they attach themselves to the ionised acceptor sites. The resulting resistivity of drifted material is well above $100,000 \mathrm{ohm}^{-\mathrm{am}}$ and with the application of 500 volts, it is fully depleted with over bias shared between the two contacts. Fig 2 shows $x$-ray absorption versus energy for $3 \mathrm{~mm}$ of active silicon.

\section{Detector contacts}

The work on surface barrier detectors led to a range of different contacts that can be used for both the entrance and rear contact. The entrance contact should have high $\mathrm{x}$-ray transmission, high reflectance to infrared (particularly for germanium) and be non-injecting. All early Si(Li) detectors employed a gold entrance contact but when the 'windowless' range of detectors was developed, a better contact was required to see the very soft $x$-rays more effectively. Contacts with lower $Z$ and density were tried - for example, palladium, nickel, cobalt, silver and others. In today's spectrometer the contact is chosen for the application.

\section{Ionisation and charge flow in the detector}

The incident $x$-ray loses energy by two main processes depending on energy. At low energies (below $10 \mathrm{keV}$ ), the photoelectric effect dominates. Above this energy the interaction is increasingly more by Compton scatter and at $80 \mathrm{keV}$ the two processes have equal probability. The Compton scatters produce photons of reduced energy, which in turn will either Compton scatter or be absorbed photoelectrically. It is preferable to have photoelectric interactions because the photon energy is transferred to a number of electrons in a smalllocalised volume.

Compton scatter increases the probability of interactions taking place close to the edges of the silicon and some photons may escape. This will increase the background since the energy of the Compton scattered electron will be absorbed. Whatever the energy loss process, it will eventually result in an energetic electron

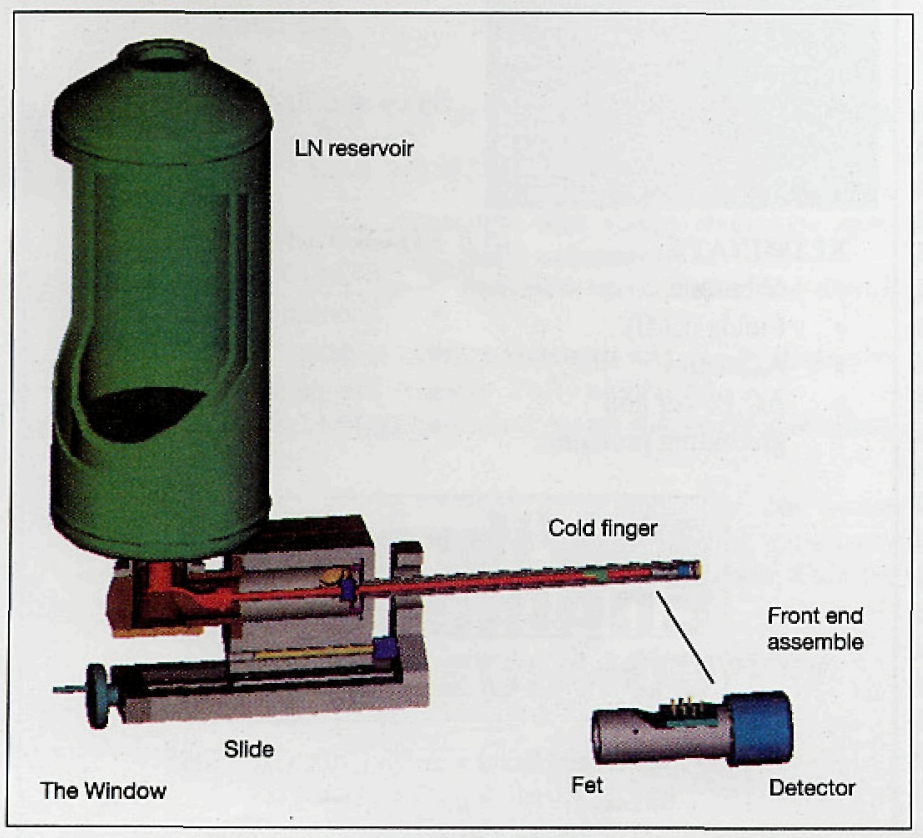

Fig. 4: The crystat and an excited silicon atom. The silicon will return to the ground state by the emission of a K-shell x-ray or an Auger electron. It is possible to view the process as the creation of two overlapping charge spheres, one due to the more energetic photoelectrons and one due to the Auger electrons.

This charge is ideally collected by the electric field and delivered to the contacts for measurement in the external circuit. In practice this occurs, except for the cases where the energetic electrons are lost directly to a contact or the charge is trapped. These charge loss mechanisms explain the shapes in the background as seen in Fig 3 .

\section{The cryostat}

The silicon detector is operated at low temperatures to reduce its reverse diode leakage current and, hence, any shot noise associated with this current. Provided the leakage current can be reduced below $0.01 \mathrm{pA}$, the noise is negligible. The cryostat design includes a cold finger, a reservoir for the liquid nitrogen, an entrance window to allow $\mathrm{x}$-rays to enter and the internal first stage of the preamplifier that again needs operating at low temperatures.

\section{The window}

The $\mathrm{x}$-rays enter the cryostat through a radiation thin window. In many instances this is a rugged thin beryllium foil ( 5 to $8 \mu \mathrm{m}$ thick). For efficient transmission of $\mathrm{x}$-rays much below $1.0 \mathrm{keV}$, a thinner window is required. Windows made from polymers on a silicon grid give very good transmission down to $100 \mathrm{eV}$. Similarly, windows made from diamond or boron nitride can be used. All these windows show the effects of absorption edges and losses to a grid structure but they are surprisingly strong, supporting atmospheric pressures and are resistant to radiation damage. The transmission curves are shown in Fig 5.

\section{Field effect transistors}

The charge developed in the silicon sensor flows to its contacts and then to the gate connection of a field effect transistor (Fet), the first stage of a low noise charge sensitive preamplifier. The development of Fets with low noise and on-chip electronic restoration of charge was critical to the performance of the modern x-ray detector.

The ideal Fet is one where the cooled noise is very low and the input capacity matches the capacity of the detector. The low capacity is achieved using the four-terminal geometry and the low noise is achieved by minimising the number of defects in the Fet channel. Each photon develops a small charge package that appears at the input of the Fet in the presence of the detector's leakage current. The charge package can be viewed as a sudden transient on the output due to leakage current

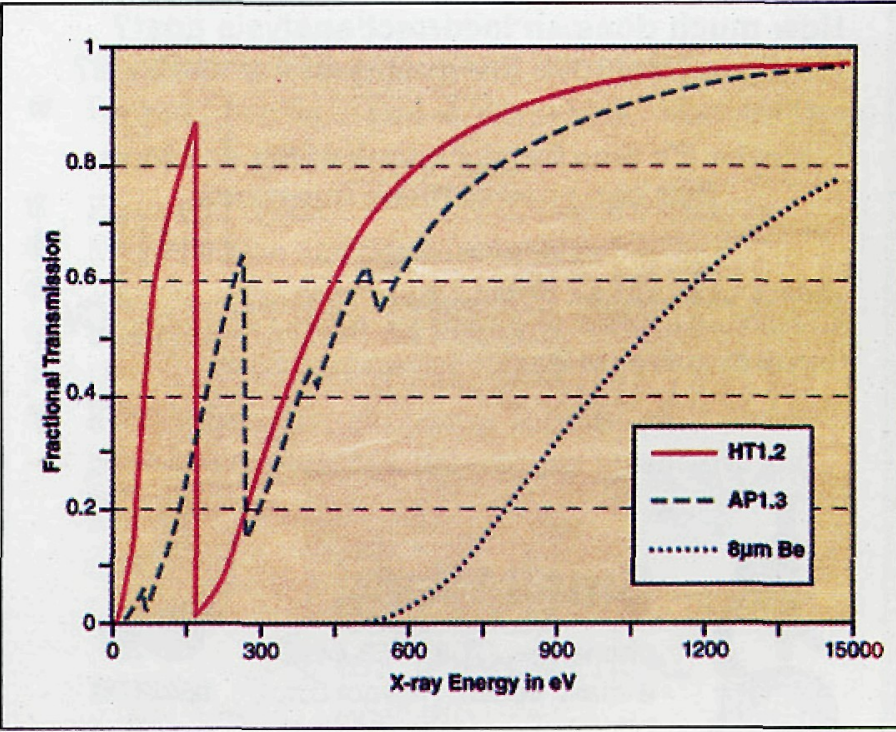

Fig. 5: Typical window transmission curves 
Semiconductor X-Ray Detectors

Continued from previous page

Eventually the response will reach a discriminator level and the output is returned to its starting position by removing the total input charge. This can be done by a variety of different methods, all having their own special properties. Discharging via a resistor is both noisy and rates limiting. Discharging using pulsed optical technology is successful but adds another component to the low temperature circuits and affords the possibility of light leakage and its after effects. Transistor restore, the latest technique is the most successful because it is fast and uses the minimum amount of dielectric materials. The modern Fet has on chip technology to make it free from dielectric noise and microphony, and it easily and efficiently attains its optimum operating temperature (approximately $145^{\circ} \mathrm{K}$ ).

\section{Summary}

The modern detector has come a long way since the first measurements in the late sixties with resolutions of $700 \mathrm{eV}$ and peak to back-grounds of 500:1. Today's silicon detector is capable of better than $130 \mathrm{eV}$ resolution with a peak to background of greater than 20,000:1. Its contacts can be matched to the application minimising fluorescent effects and the appearance of photoelectrons in a critical region. The thickness can be selected so that in the application the proportion of background due to Compton scatter can be reduced in the measurement range of interest. Also, in XRE, the detector can be made reasonably transparent to $x$-ray tube characteristic lines. In certain measurements it may be an advantage for the detector to transmit the higher energy $x$-ray. In the future the combined benefits from several semiconductors will be used to advantage as will the application of novel cooling techniques.

In recent years the cycle of development has produced new x-ray detec- tors. PIN diodes make use of shallow depleted structures with diffused contacts designed for optical detection. The $x$-ray response in a limited energy range is very good (<165 eV with a peak to background of 1000:1). Similarly, the Drift Detectors, another shallow structure, can be pixellated to produce devices with large areas, preserving good resolution ( $<140 \mathrm{eV}$ with peak to backgrounds of 1000:1). Shallow depletion depths and poorer peak to backgrounds limit the performance of both structures. They also do not have the benefit of the specially designed thin electrodes developed for the standard semiconductor $\mathrm{x}$-ray detectors. However, good low energy performance has recently been demonstrated with cooled CCD arrays and PN CCD structures, developed for position and energy measurements in $\mathrm{x}$-ray astronomy.

Using the $\mathrm{Si}(\mathrm{Li})$ as today's standard to compare against, all these detectors have a place in the wide field of research and industrial measurements. The theory of operation of all these devices is similar; the differences often explained by variations in their electrodes and depletion depths. The history goes back to surface barrier structures designed for nuclear physics.

This article originally appeared in Microscopy and Imagnig News (UK), July 1998 issue.

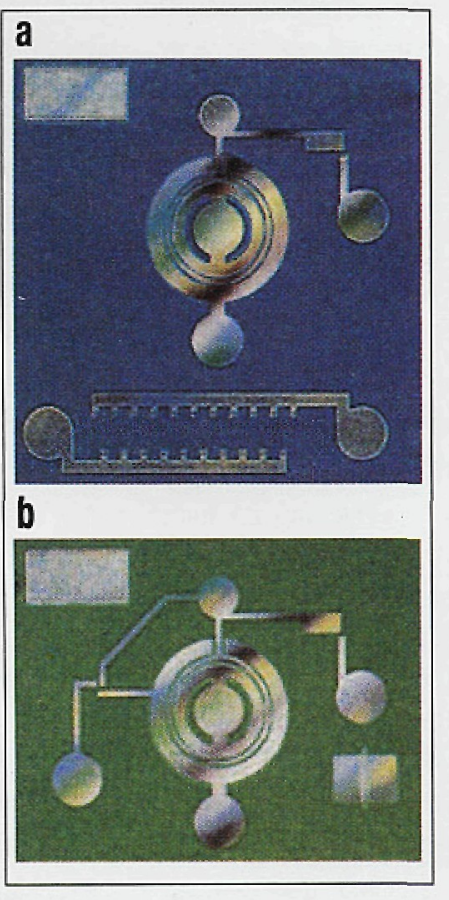

Fig. 6: (a) Fet with on-chip heater and feedback cpacitor. (b) Fet with transistor restore.

\section{Take the following microanalysis quiz}

\begin{abstract}
What is the thickness of my film? Does the beam penetrate that particle? What is the best $\mathrm{kV}$ to use for this sample? How wide is the beam in my E-SEM? How much does an incorrect analysis cost? How can I improve the quality of my analysis?

\section{Maybe it's time to take a look at the software that can answer these questions}

\section{Electron Flight Simulator}

Analysis Simulation and Modeling Software for Windows

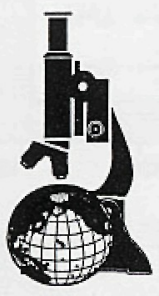

\section{Small World}

phone/fax: (703) 849-1492 e-mail: dchernoff@aol.com http://www.small-world.net

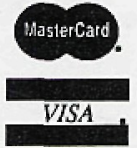

\section{Does your image look like this?}

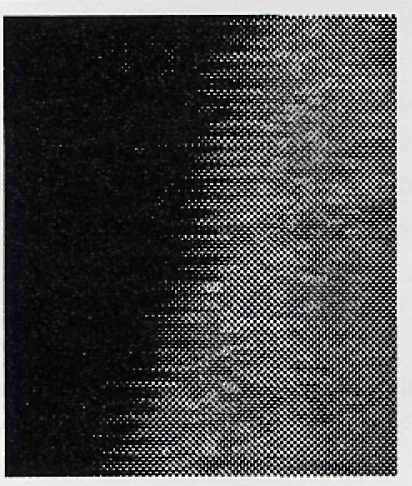

\section{ELIMINATE}

- Vibration

- Fields (EMI)

- Acoustics

- $\mathrm{AC}$ Power and grounding problems
Let us solve your disturbance problems.

Site Surveys

All types of equipment.

Site Design

Sales and Service, Spicer ${ }^{\mathrm{TM}}$

Cancellation Systems

\section{EQUIPMENT}

- SEM/TEM

- Scanning Auger / ESCA

- e-beam

- Steppers
- $\mathrm{SPM} / \mathrm{AFM}$

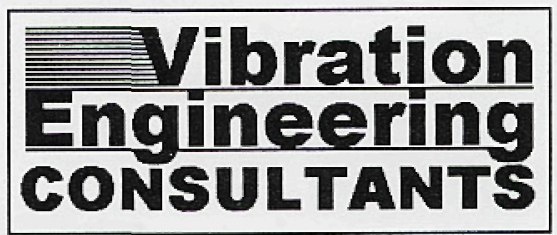

4555 Paul Sweet Road • Santa Cruz, CA 95065

$831-465-9189 \bullet$ fax 831-465-9341

www.vibeng.com 


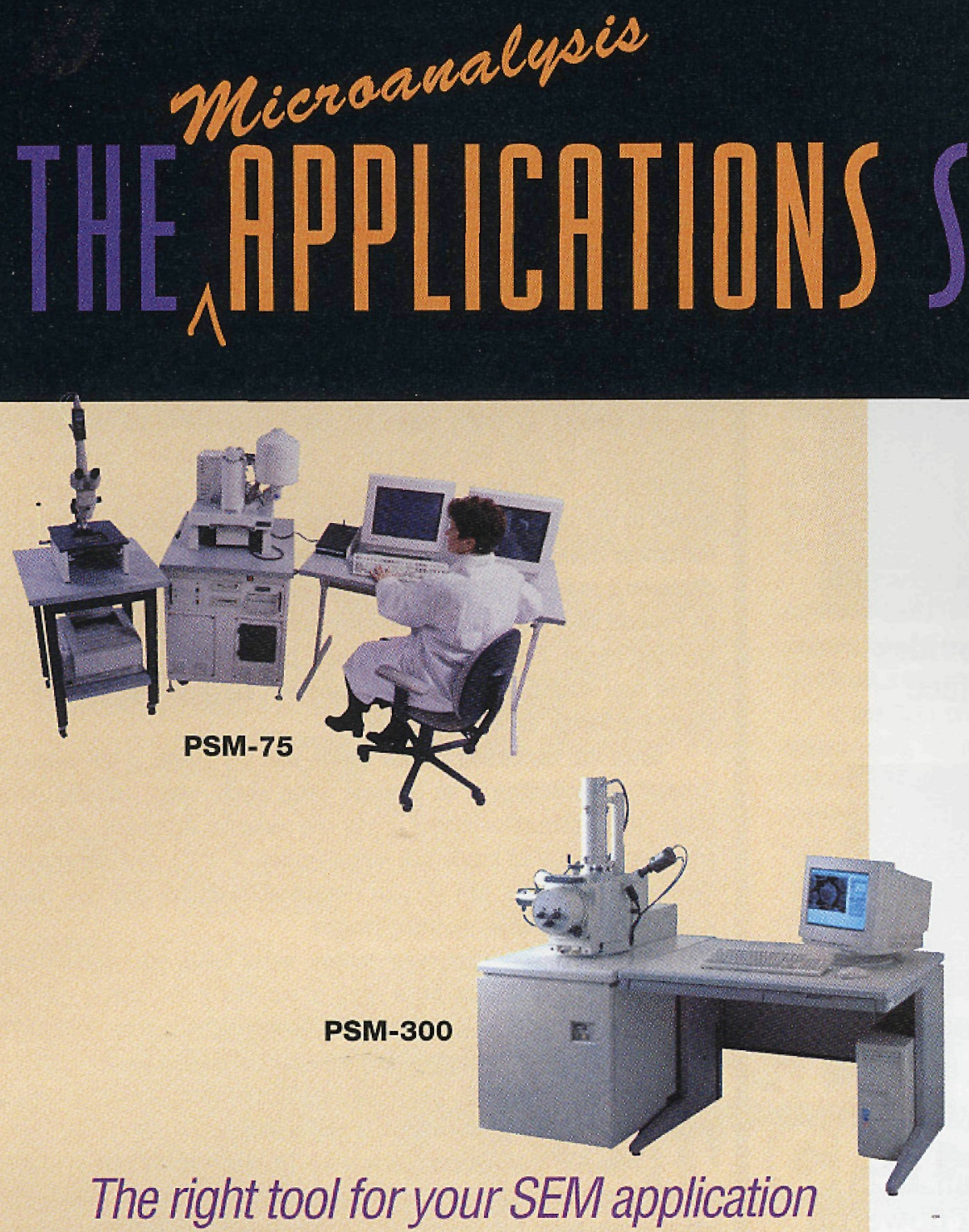

When you invest in an SEM, you need an instrument that will most cost effectively handle your unique analytical requirements... like the PSM-75 or PSM-300.

Powerful, highly functional, yet simple-to-use, RJ Lee Instruments' SEMs are easily customized for specific applications with the addition of hardware options or software programs.

- Fully-automatic variable pressure capabilities can be retrofitted on-site

numerous application tools are available, including:

- Automated Inclusion Analysis

- Automated Mineral Analysis

- Automated Fiber Analysis

- Automated Particle Analysis

- Automated GSR Analysis

- Preview \& Relocation (P\&R) Station

Whether you are an experienced SEM user seeking to supplement or replace an existing SEM, or one who is exploring its potential for the first time, you will find our instruments powerful, practical and versatile tools, fully meeting your needs at an affordable price.

\section{RJ Lee Instruments has the versatility to fulfill your unique requirements}

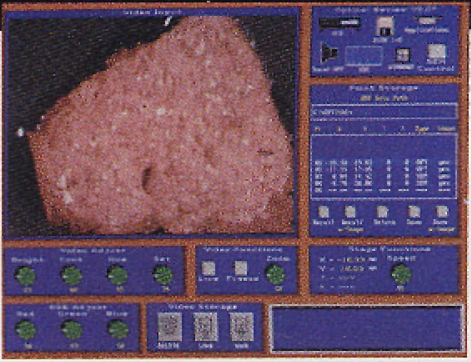

Optical Preview and SEM Comparison

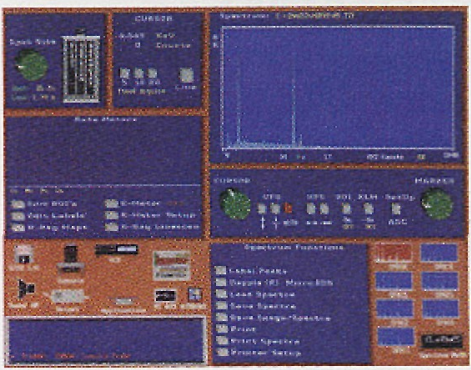

Integrated EDS X-ray Analysis

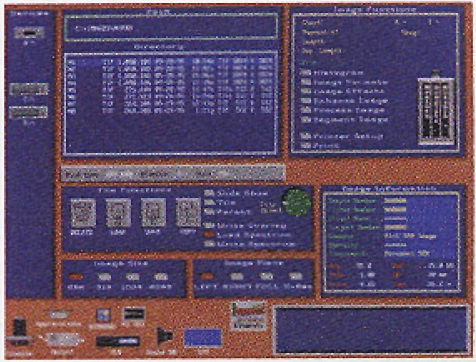

Archiving

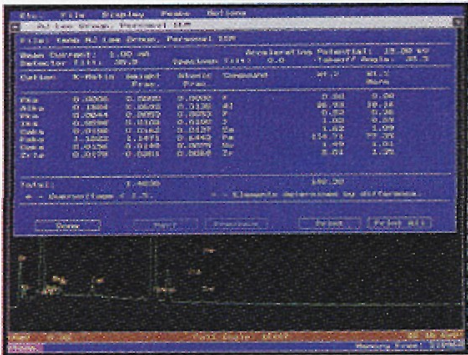

Quantitative Feature Analysis Booth 50 


\section{EDS Upgrades}

Upgrade your existing EDX system with the world leader in affordable EDX upgrades.

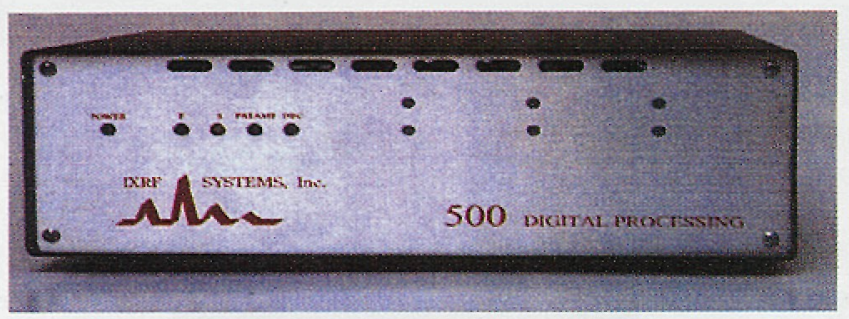

Our new digital pulse processor provides increased throughput and will interface to most EDS detectors.

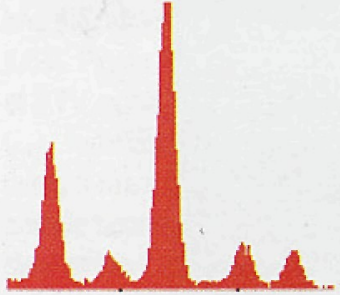

Precise Standardless quantitation for even complex matricies

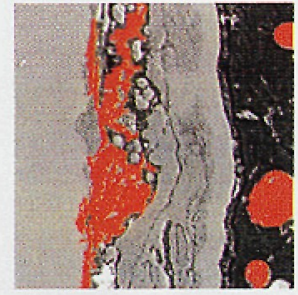

"Active" digital imaging, linescans, and $\mathrm{x}$-ray mapping.
Combining digital imaging with our EDS system produces a very simple yet powerful Microanalysis system.

\section{IXRF has established it-self as a "leader"} in the Microanalysis industry as most of the major EDS companies convert from analog to digital systems.

- Hundreds of systems installed

- Easy to use Windows 95 or NT

- Each system comes complete with Pentium PC

- Installation and training at your site

- New detectors available

- Morphology and Feature analysis

- Automatic Point Analysis

- Quantitative Mapping and Linescans

IXRF Systems, Inc. 15715 Brookford Dr. Houston, TX 77059

\section{Down With Magnification: The Micron Marker Rules! Joe Geller \\ Geller Microanalytical Laboratory}

With today's current wave of quality consciousness, our quality control people tell us we MUST calibrate our instruments using standards that are traceable to the national laboratories (NIST - National Institute of Standards and Technology in the U.S.,. NPL - National Physical Laboratory in the UK, and others). But, is it really magnification that should be calibrated?

While recently walking around the exhibit floor at the Microscopy \& Microanalysis ' 98 Conference, I noticed the large SEM image display screens that now present our highly magnified specimens. Almost all vividly show a ${ }_{0}^{0}$ micron (using SI units this should be a "micrometer") marker as well as the magnification. No doubt the accuracy is within the $\pm 3 \%$ that is commonly quoted by the manufacturers. So what's the problem? Maybe this is picky. The micrometer marker is probably within the stated accuracy but the magnification displayed is really only meant for the final image (which was probably recorded on Polaroid film). However, if the display screen is larger than the size of the recorded image, the magnification should be appropriately scaled. On the exhibit floor magnification errors increased with the display screen size. The bigger the display, the larger the error.

Since the micrometer marker scales with the image, it will always be "correct". Magnification is an absolute value which, when recorded on the image, will be correct for images at only one particular image size. Unless you are using the Polaroid image size (approximately $4^{\prime \prime} \times 5^{\prime \prime}$ ), and that image will forever stay that size, turn off your magnification display and only use the micrometer marker when recording images.

To calibrate your micrometer marker, either follow your manufacturer's recommendation or ask us for a copy of our free magnification calibration resource guide (tel.: (978)887-7000 or email: Jg@gellermicro.com). Your SEM service engineer can perform the fine adjustments to bring the instrument into calibration. They prefer to use your own trusted standard. That way the engineers are free of liability if theirs is not traceable

A new ASTM (American Society for Testing and Materials) "Standard Practice! for Calibrating the Magnification of a Scanning Electron Microscope", which is now being balloted by the E-4 Metallography Committee, addresses these points. When available, it will be named E776-XX where the last two digits reflect the year of balloting for this standard. What will happen to the standard's name if it's approved in Y2K?

\section{$=-m=-m=-m$ \\ | HISTOLOGY TECHNICIAN}

Riverside Regional Medical Center seeks an experienced Histology Technician with Medical Electron Microscopy experience to work full-time in our laboratory department in the Histology Department. The hours are Monday - Friday, 8:00 a.m. to 4:30 p.m. Candidates must be able to work independently and with several physicians. Apply to:

Riverside Regional Medical Center Human Resources Department $500 \mathrm{~J}$ Clyde Morris Blvd Newport News, VA 23601

Tel.: (757)594-2025 $\uparrow$ Fax: (757)594-3864

Riverside Regional Medical Center is a Aflirmative Action/Fqual Opportunity Employer 